\title{
Comparison of the VeC-HF and VeC-HB function based on the Normal Posture: H-FB
}

\author{
Jeong-lae Kim ${ }^{1}$, Kyu-sung Hwang ${ }^{2}$ and Yong-seok Nam ${ }^{3 *}$ \\ ${ }^{1}$ Department of Biomedical Engineering Eulji University, Seoul, Korea \\ ${ }^{2}$ Department of Funeral Science Eulji University, Seoul, Korea \\ ${ }^{3}$ Department of Anatomy, Catholic institute for Applied Anatomy Catholic University, Seoul, Korea \\ ${ }^{1}$ jlkim@eulji.ac.kr, ${ }^{2} h k s @ e u l j i . a c . k r,{ }^{3}$ boffin@unitel.co.kr \\ *Corresponding author should be addressed to Kyu-sung Hwang, hks@eulji.ac.kr
}

\begin{abstract}
To compare Vestibular-CNS function on the HF and HB condition confirmed to balance normal state of a posture. The vestibular and CNS displacements analyzed at the five ranges from the H-FB: Head of forward and backward. Vestibule-CNS function (VeC) shows the vestibular variation appear to through the CNS from the spinal cord. The average different outputs obtained greatest the perturbations: VeHB-CNO-B displacement at 10.88 \pm 2.71 , VeHB-CNC-B displacement at 10.76 2.68 , VeHB-CNO-A displacement at 10.077 \pm 2.33 , VeNC-CNC-A displacement at 10.58 \pm 2.72 . Our results will be to show an impact of at least postural control by the H-FB function. CHF-CNC-A and CHB-CNC-A displacement of the moment identified to inertia from CNS. The Vestibular HF-HB was affected to maintain for the balance by the CNS of HF-HB.
\end{abstract}

Keywords: CNS; VeC-HB, VeC-HF, H-FB; Normal Posture

\section{Introduction}

The precise role of vestibular afferent input in posture and balance control is unclear, especially for voluntary movement $[1,2]$. The ability to maintain posture and balance of the human was to voluntary movements on the station. Moreover, loss of balance was a clinically important problem, as falls are a primary cause of injury and accidental death in older adults [3].

Maintaining and controlling Posture balance in normal state maintains generally to detect the physical condition through peripheral sensory system, that is used the information provided by the vestibular system [4]. The afferent information must be processed and interpreted correctly by the central nervous system(CNS) through spinal, brainstem reflexes and higher order structures [5, 6].

Also a suggested body of postural variability or smaller scale postural fluctuations may improve overall postural pliability and stability [7]. Essentially, it has been suggested that small scale postural fluctuations allow the individual to investigate the dynamically physical condition so that possibilities for action are assessed and movements more clearly generated $[8,9]$. Lack of postural variability, in contrast, can be independent of constructive hardness [10].

Therefore, the postural method required to maintain balance differ from a movement. Far fewer studies have examined balance control when to sway the control of whole body centre of mass (COM) and to reveal how body are coordinated to stabilize upright state [11].

In this study, correlation of the vestibular-CNS system for balance based desired physical condition was compared to a physical signal in the normal state. Body condition was consist 
of a physical parameters for the normal eye open(NO) and close(NC). This system was used to set the stability for the evaluation of the biomechanical information and to control by the data processing system.

\section{Related Works}

\subsection{Physical Condition of Ve-HF and Ve-HB}

The postural balance system consists of physical signal on the subsystems such as included the vestibular and CNS [12]. Sway is interpreted as the reflection of noise and regulatory activity within postural. However it is not explained postural sway occurs during stance, and the potential characteristics of a change in sway behavior represent a lacked balance. There is in fact little scientific agreement on what sway actually represents. The COP displacement is the controlling variable in biomechanical terms, and reflecting the responses generated by the CNS to maintain control over the COM [13].

A specific postural response stage, the activation to stabilize the body COM is closely tuned to the biomechanics of the perturbation, especially, direction. When the support surface is translated in each of many directions in the horizontal plane, sensory across the body are activated by an amount related to the direction of the perturbation. Therefore, a different set of the body is activated in response to a forward perturbation versus a backward perturbation [14-17]. The parameter relies on vestibular condition of head forward(HF) and backward(HB) relationships to measure physical condition. Physical Ve-C condition flowchart collects to normal state of the body and to experiment on the parameter as shown Figure 1.

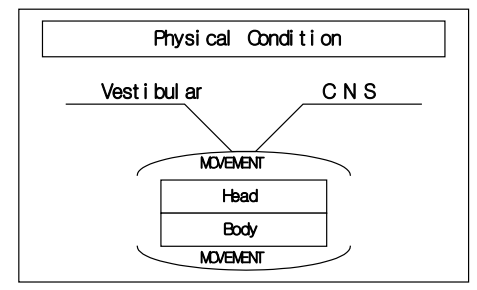

Figure 1. Structure of Physical Ve-C condition

\subsection{Component of CNS and Body Balance}

The CNS must coordinate motion across many an organ using sensory information provided to maintain upright stance by vestibular systems. Body balance is a complex process requiring integration of the sensory information and implement of appropriate postural responses. Also postural coordination and the role of different sensory systems change for appropriately stable balance control under the CNS conditions [11].

The body balance has controlled from simple perturbations and imposed by a volitional movement, examined balance control during continuous perturbations. During continuous perturbations using a repeated to support some movement, perturbations become predictable the postural control, although balance becomes more manageable. Human foresee the characteristics of perturbations and their destabilizing effects within the first few cycles of perturbation, and then to put their balance control system to minimize these effects [19]. During continuous perturbations, movements of the head segment may be minimized to provide a stable condition for the vestibular sensory systems [18]. The motion of the body and head becomes increasingly parts as the perturbation frequency increases yielding the impression that different postural control are organizationally acquired [19, 20]. Physical Ve- 
C condition flowchart collects to normal state of the body and to experiment on the parameter as shown Figure 1.

\section{System Design: Vestibular-CNS System}

\subsection{Preprocessing}

The proposed vestibular-CNS system mainly showed the monitoring subjects that had a posture balance for head movement. There are normally showed the body condition is comfortable state, when the physiological condition becomes normal and stable.

\subsection{Physiology of Vestibular-CNS System}

The CNS appeared through the body movements on the center state, the reciprocal relation between the vestibular of semicircular canals arrowed dimensional head movements and the CNS, as the vector sum of components expressed on the physical condition. Alignment of the semicircular canals is roughly associated by the multi- direction of the CNS. The organizational scheme provides an appropriate mechanism for spatial alignment of the reflexes. Because of their mechanical properties, the canals integrate this additional signal over the some alteration range of head movements. And then Vestibular CNS system was to effect on force of inertia [21].

Postural control is complicated process demanding integration of the sensory information and implementation of appropriate postural condition. To maintain stance, the CNS must coordinate motion across using sensory information provided by vestibular systems [22]. The COM displacement is desired to be minimized by the CNS through adjustments in COP either through forward [23] or backward condition [24].

The architecture of the proposed system was depicted in Figure 2. System architecture of a Ve C-HF \& Ve C-HB system showed physical signal for normal state on the stance. Data sheets acquired a data control system signal processing. The equipment was designed as data acquisition system (PXI-6251 DAQ) and Signal processing system(PXI-1409, NI., USA) [2527].

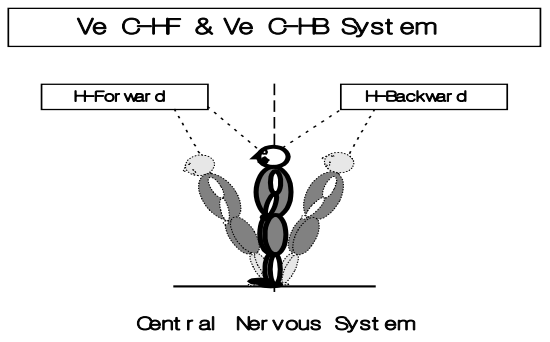

Figure 2. System Architecture of a Ve C-HF \& Ve C-HB Presented Physical Signal for Normal State by the Platform. Mean and S.D. for Comparison between CNS and Vestibular Displacement of Data in the Head Sway

\section{Experimental Results}

\subsection{Experimental Results}

The subsection gives an analysis for our proposed system these parameters, and the condition is dependent on each other. In order to further understand how to use these 
parameters to design each parameter. Several data are performed and results are explained as follows.

\section{Comparison of Ves_NO_B and CNS_NO_B : Avg HF- $\mu_{C N O B}$, Avg HB- $\mu_{C N O B}$}

The comparison of vestibular and CNS values at the five ranges of position generally appeared a great variation at all condition based on the CNS of normal eye open state(NO)(Figure 3). There showed an experiment record of the displacement values on the CNS of NO and Vestibular of NO condition from body of movement before; such as VeNOCNO-B, VeHF-CNO-B, VeHB-CNO-B, CHF-CNO-B and CHB-CNO-B. Absolute VeHBCNO-B value was a great displacement across all ranges, secondly was VeHF-CNO-B value and the whole range was from 0.137 to 10.88. These tests displayed that VeHB-CNO-B displacement was greatest at $10.88 \pm 2.71$ perturbations, secondly VeHF-CNO-B was 9.66 \pm 2.105 . CHB-CNO-B displacement was smallest at $0.13 \pm-0.009$ perturbations, second to last CHF-CNO-B was $0.14 \pm-0.12$ perturbations. The more average of the differences presented between Avg HF- $\mu_{\mathrm{CNOB}}$ and Avg HB- $\mu_{\mathrm{CNOB}}$. Comparison data analyzed significant relationship of range displacement between the Vestibular of HF-HB and the CNS of HF-HB. Correlations between Vestibular and CNS displacement were more across all range of perturbation (ranging from 0.13 to 10.88) (Table 1 ).

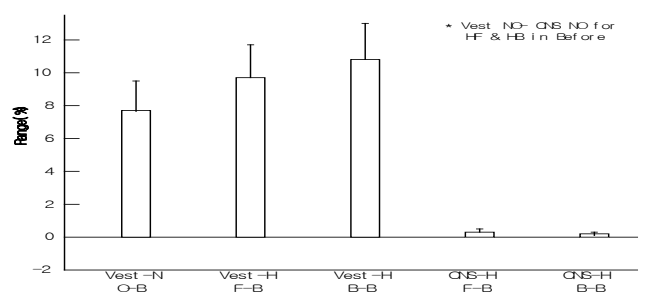

Figure 3. Avg. and S.D for

Comparison between Vestibular and CNS Range of Data of the Normal

Posture in the before Movement (NO Condition of Vest NO-CNS NO_B).

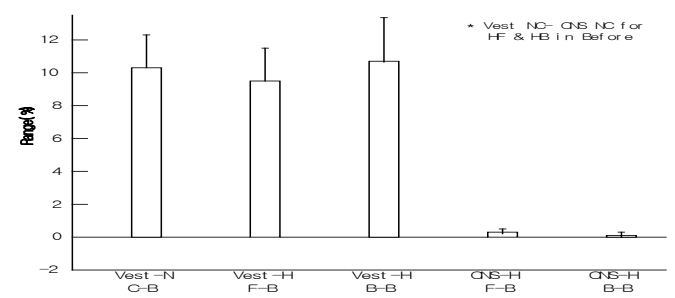

Figure 4. Avg. and S.D for Comparison between Vestibular and CNS Range of Data of Data of the Normal Posture in the before Movement (NC Condition of Vest NCCNS NC_B).

\section{Comparison of Ves_NC_B and CNS_NC_B : Avg HF- $\mu_{C N C B}$, Avg HB- $\mu_{C N C B}$}

The comparison of vestibular and CNS values at the five ranges of position generally appeared a great variation at all condition based on the CNS of normal eye close state(NC)(Figure 4). There showed an experiment record of the displacement values on the CNS of NC and Vestibular of NC condition from body of movement before; such as VeNCCNC-B, VeHF-CNC-B, VeHB-CNC-B, CHF-CNC-B and CHB-CNC-B. Absolute VeHBCNC-B value and VeNC-CNC-B was a great displacement across all ranges and the whole range was from 0.017 to 10.76 . These results displayed that VeHB-CNC-B displacement was greatest at 10.76 \pm 2.68 perturbations, secondly VeNC-CNC-B was 10.34 \pm 2.46 . CHB-CNC-B displacement was smallest at $0.17 \pm-0.045$ perturbations, second to last CHF-CNC-B was $0.02 \pm 0.086$ perturbations. The more average of the differences presented between Avg HF$\mu_{\mathrm{CNCB}}$ and Avg HB- $\mu_{\mathrm{CNCB}}$. Vestibular of HF-HB and the CNS of HF-HB was compared to slightly significant relationship at the head movement. The VeHB-CNC-B appeared to sway the VeNC-CNC-B state on the NC condition. Correlations between Vestibular and CNS displacement were more across all range of perturbation (ranging from 0.02 to 10.76) (Table $1)$. 
Comparison of Ves_NO_A and CNS_NO_A : Avg HF- $\mu_{C N O A}$, Avg HB- $\mu_{C N O A}$

The comparison of vestibular and CNS values at the five ranges of position generally appeared a great variation at all condition based on the CNS of normal eye open state(NO)(Figure 5). There appeared an experiment record of the displacement values on the CNS of NO and Vestibular of NO condition from body of movement after; such as VeNOCNO-A, VeHF-CNO-A, VeHB-CNO-A, CHF-CNO-A and CHB-CNO-A. Absolute VeHB$\mathrm{CNO}-\mathrm{A}$ value was a great displacement across all ranges and the whole range was from -0.02 to 10.077. These results displayed that VeHB-CNO-A displacement was greatest at $10.077 \pm 2.33$ perturbations. CHF-CNO-A displacement was smallest at $-0.02 \pm-0.029$ perturbations, second to last CHB-CNO-A was $0.05 \pm-0.02$ perturbations. The more average of the differences presented between Avg HF- $\mu_{\mathrm{CNOA}}$ and Avg HB- $\mu_{\mathrm{CNOA}}$. Vestibular of HF$\mathrm{HB}$ and the CNS of HF-HB was compared to slightly significant relationship at the head movement. The VeHB-CNO-A appeared to sway the VeNO-CNO-A state on the NO condition. Correlations between Vestibular and CNS displacement were more across all range of perturbation (ranging from -0.02 to 10.07) (Table 1 ).

The slightly greater differences of the CNS of NO parameter confirmed relationship range of the Vestibular of HF-HB at the head movement. CHF-CNO-A displacement of the moment showed a significant effect of CNS range that identified to inertia from main center (ranging 0.02). Then CNS-HF affected to maintain in balance.

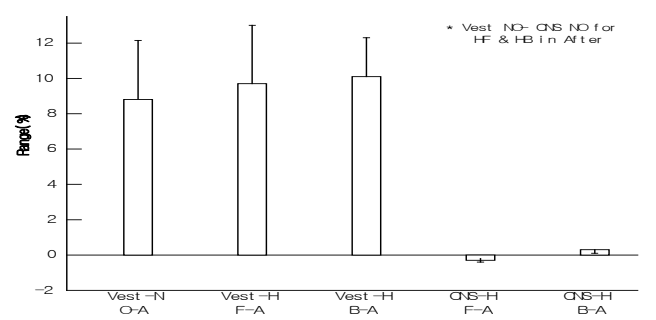

Figure 5. Avg. and S.D for Comparison between Vestibular and CNS Range of Data of the Normal Posture in the after Movement (NO Condition of Vest NO-CNS NO_A)

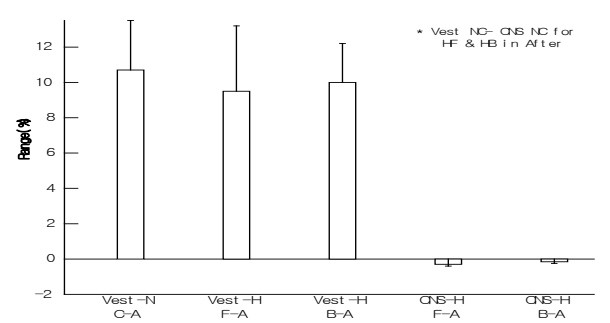

Figure 6. Avg. and S.D for Comparison between Vestibular and CNS Range of Data of Normal Posture in the after Movement (NC Condition of Vest NC-CNS NC_A)

Table 1. Avg. and S.D of Range on the Response on the Four Different Condition: VeNO $\mu_{\mathrm{CNO}-\mathrm{B}}$, VeNC $\mu_{\mathrm{CNC}-\mathrm{B}}$, VeNO $\mu_{\mathrm{CNO}-\mathrm{A}}$ and VeNC $\mu_{\mathrm{CNC}-\mathrm{A} \text {. }}$ Presentation of Head Moving of Eye Open(NO) and Eye Close(NC)

\begin{tabular}{llllll}
\hline Average $\mu$ & VeNO $\mu_{\text {Avg }}$ & VeHF $\mu_{\text {Avg }}$ & VeHB $\mu_{\text {Avg }}$ & CHF $\mu_{\text {Avg }}$ & CHB $\mu_{\text {Avg }}$ \\
\hline$\mu_{\text {CNO-B }}$ & $7.62 \pm 1.89$ & $9.66 \pm 2.11$ & $10.88 \pm 2.71$ & $0.14 \pm 0.12$ & $0.13 \pm-0.009$ \\
\hline Average $\mu$ & VeNC $\mu_{\text {Avg }}$ & VeHF $\mu_{\text {Avg }}$ & VeHB $\mu_{\text {Avg }}$ & CHF $\mu_{\text {Avg }}$ & CHB $\mu_{\text {Avg }}$ \\
\hline$\mu_{\text {CNC-B }}$ & $10.34 \pm 2.46$ & $9.54 \pm 2.06$ & $10.76 \pm 2.67$ & $0.02 \pm 0.08$ & $0.02 \pm-0.045$ \\
\hline \multicolumn{7}{c}{} \\
\hline Average $\mu$ & VeNO $\mu_{\text {Avg }}$ & VeHF $\mu_{\text {Avg }}$ & VeHB $\mu_{\text {Avg }}$ & CHF $\mu_{\text {Avg }}$ & CHB $\mu_{\text {Avg }}$ \\
\hline$\mu_{\text {CNO-A }}$ & $8.84 \pm 3.02$ & $9.62 \pm 3.39$ & $10.07 \pm 2.33$ & $-0.02 \pm-0.03$ & $0.056 \pm-0.02$ \\
\hline \multicolumn{7}{c}{} & & & \\
\hline Average $\mu$ & VeNC $\mu_{\text {Avg }}$ & VeHF $\mu_{\text {Avg }}$ & VeHB $\mu_{\text {Avg }}$ & CHF $\mu_{\text {Avg }}$ & CHB $\mu_{\text {Avg }}$ \\
\hline$\mu_{\text {CNC-A }}$ & $10.58 \pm 2.72$ & $9.48 \pm 3.26$ & $9.93 \pm 2.20$ & $-0.167 \pm-0.15$ & $-0.089 \pm-0.14$ \\
\hline
\end{tabular}




\section{Comparison of Ves_NC_A and CNS_NC_A : Avg HF- $\mu_{C N C A}$ Avg HB- $\mu_{C N C A}$}

The comparison of vestibular and CNS values at the five ranges of position generally appeared a great variation at all condition based on the CNS of normal eye close state(NC)(Figure 6). There appeared an experiment record of the displacement values on the CNS of NC and Vestibular of NC condition from body of movement after; such as VeNCCNC-A, VeHF-CNC-A, VeHB-CNC-A, CHF-CNC-A and CHB-CNC-A. Absolute VeNCCNC-A value was a great displacement across all ranges and the whole range was from 0.089 to 10.58 . These results displayed that VeNC-CNC-A displacement was greatest at $10.58 \pm 2.72$ perturbations. CHB-CNC-A displacement was smallest at $-0.089 \pm-0.149$ perturbations, second to last CHF-CNC-A was -0.16 \pm-0.158 perturbations. The more average of the differences presented between Avg HF- $\mu_{\mathrm{CNCA}}$ and Avg HB- $\mu_{\mathrm{CNCA}}$. The Vestibular of HF-HB and the CNS of HF-HB was to inertia slightly significant relationship at the head movement. Vestibular and CNS displacement were a variety affects all range of perturbation (ranging from -0.089 to 10.58 ) (Table 1 ).

The slightly greater differences of the CNS of NC parameter confirmed relationship range of the Vestibular of HF-HB at the head movement. CHF-CNC-A and CHB-CNC-A displacement of the moment showed a significant effect of CNS range that identified to inertia from main center (ranging -0.09). The Vestibular HF-HB was affected to maintain for the balance by the CNS of HF-HB. The HF and HB was affected a greatly to sway Vestibular and CNS state on the NC condition

\subsection{Performance Evaluations}

Comparision on the vestibular-CNS system presented the results of the normal state by the EO and EC. On the posture balance showed a difference consequence by the body condition according to exercise after and before.

Balance control during stance depends on the ability to keep the control of base on the COM of the body $[28,29]$. This study provides a definite analysis of the some control to maintain stay on a stable platform. To compare on the vestibular-CNS system, presented the results of the normal state by the eye open and eye close. On the posture balance showed a difference consequence by the body condition according to exercise after and before. The important findings come out a displacement of the head exceeded control of the body COM.

A number of studies of postural control during translational perturbations have suggested that stabilization of the head is the primary goal of postural control [30, 31]. In our study, head movement decreased as the perturbation increased displacement remained greater than that of whole body COM across all condition.

Their research presented to contribute by the source of sensory information to postural control [32, 33]. We showed a critical increase in the displacement of CNS and Ve-HF-HB function during head movement [34, 35].

In our study, firstly the displacement values on the CNS of NO and Vestibular of NO condition from body of movement before, more average of the differences presented between VeHF- $\mu_{\mathrm{CNOB}}$ and VeHB- $\mu_{\mathrm{CNOB}}$. There showed significant relationship of range displacement between the Vestibular of HF-HB and the CNS of HF-HB.

Secondly, there showed the displacement values on the CNS of NC and Vestibular of NC condition from body of movement before, more average of the differences presented between VeHF- $\mu_{\mathrm{CNCB}}$ and VeHB- $\mu_{\mathrm{CNCB}}$. The VeHB- $\mu_{\mathrm{CNCB}}$ showed to sway weakly the VeNC- $\mu_{\mathrm{CNCB}}$ state. Vestibular of HF-HB and the CNS of HF-HB was compared to slightly significant relationship at the head movement. 
Thirdly, there showed the displacement values on the CNS of NO and Vestibular of NO condition from body of movement after, more average of the differences presented between VeHF- $\mu_{\mathrm{CNOA}}$ and VeHB- $\mu_{\mathrm{CNOA}}$. Vestibular of HF-HB and the CNS of HF-HB was compared to slightly significant relationship at the head movement. The VeHB- $\mu_{\mathrm{CNOA}}$ appeared to sway the VeNO- $\mu_{\mathrm{CNOA}}$ state on the NO condition. To compare Vestibular and CNS displacement were more across perturbation range of from -0.02 to 10.07. The CNS of NO parameter confirmed slightly greater differences of the Vestibular of HF-HB at the head movement. $\mathrm{CHF}-\mu_{\mathrm{CNOA}}$ displacement of the moment showed a significant effect of CNS, and identified to inertia from CNS. And then CNS-HF affected to maintain in balance on the NO condition.

Last, there showed the displacement values on the CNS of NC and Vestibular of NC condition from body of movement after; more average of the differences presented between Ve HF- $\mu_{\mathrm{CNCA}}$ and Ve HB- $\mu_{\mathrm{CNCA}}$. The Vestibular of HF-HB and the CNS of HF-HB was to inertia slightly significant relationship at the head movement. To compare Vestibular and CNS displacement, a variety affects presented all range of perturbation from -0.089 to 10.58 . The slightly greater differences of the CNS of NC parameter confirmed relationship range of the Vestibular of HF-HB at the head movement. CHF-CNC-A and CHB-CNC-A displacement of the moment showed a significant effect of CNS, and identified to inertia from CNS. The Vestibular HF-HB was affected to maintain for the balance by the CNS of HF-HB. The HF and HB was affected a greatly to sway Vestibular and CNS state on the NC condition.

In addition to COM displacement, our results indicated that while standing on the stance, regardless of tiny perturbation, posture balance may caused by participants reduce movements of the CNS through the anti-phase motion among the body. The COM displacement is desired to be minimized by the CNS through adjustments in the CNS either through forward or backward condition [36, 37].

\section{Conclusion}

We was defined a comparison of vestibular-CNS system on the basis of the normal state in the standing body. To compare Vestibular-CNS function on the HF and HB condition confirmed to balance normal state of a posture. The displacement values changed at the CNS and Vestibular state from body of movement by the NC-NO condition.

The average different outputs obtained greatest the perturbations: VeHB-CNO-B displacement at 10.88 \pm 2.71 , VeHB-CNC-B displacement at 10.76 \pm 2.68 , VeHB-CNO-A displacement at 10.077 \pm 2.33 , VeNC-CNC-A displacement at 10.58 \pm 2.72 . Our results will be to show an impact of at least postural control by the H-FB function.

Our results indicated that while standing on the stance regardless of tiny perturbation, participants reduce movements of the CNS by employing anti-phase motion about the body. To compare Vestibular-CNS function on the HF and HB condition confirmed to balance normal state of a posture.

In the future, this system will be express to an assessment for body balance in dynamic condition and multi-parameter to head movement within a vestibular and central nervous system.

\section{References}

[1] J. T. Inglis and J. M. Macpherson, "Bilateral labyrinthectomy in the cat: effects on the postural response to translation”, J Neurophysiol, vol.73, (1995), pp. 1181-1191.

[2] J. M. Macpherson and J. T. Inglis, "Stance and balance following bilateral labyrinthectomy", Natural and Artificial Control of Hearing and Balance”, edited by J. H. J. Allum, D. Allum-Mecklenburg, F. Harris and R. Probst, New York: Elsevier, (1993), pp. 219-228. 
[3] A. M. Minino, E. Arias, K. D. Kochanek, S. L. Murphy and B. L. Smith, “() Deaths: final data for 2000. Natl. Vital Stat. Rep., vol. 50, (2002), pp. 1-119.

[4] J. Massion, "Movement, posture and equilibrium: interation and coordination", Prog Neurobiol, vol. 38, (1992), pp. 35-56.

[5] D. A. Winter, A. E. Patla, S. Rietdyk and M. G. Ishac, "Ankle muscle stiffness in the control of balance during quit standing”, J Neurophysiol, vol. 85, (2001), pp. 2630-3.

[6] I. N. Beloozerova, M. G. Sirota, G. N. Orlovsky and T. G. Deliagina, “Activity of pyramidal tract neurons in the cat during postural correction”, J Neurophysiol, vol. 93, (2005), pp. 1831-44.

[7] R. Van Emmerik, "Van Wegen EE On the functional aspects of variability in postural control”, Exerc Sport Sci Rev, vol. 30, (2002), pp. 177-183.

[8] J. J. Gibson, “The ecological approach to visual perception”, Houghton MiZin, Boston, (1979).

[9] G. E. Riccio, "Information in movement variability about the qualitative dynamics of posture and orientation”, Newell KM, Corcos DM (eds) Variability and motor control, Human Kinematics, Champaignpp, (1993), pp. 317-358.

[10] K. Davids, S. Bennett and K. M. Newell, "Movement system variability”, Human Kinetics, Champaign, (2006).

[11] S. B. Akaram, J. S. Frank, A. E. Patla and H. J. Alulum John, "Balance control during continuous rotational perturbation of the support surface”, Gait \& Posture, vol. 27, (2008), pp. 393-398.

[12] S. O. O'Sullivan, “Assessment of motor function”, O'Sullivan SB, Schmitz TJ, eds. Physical Rehabilitation: Assessment and Treatment, 4rd ed. Philadelphia, PA: FA Davis, (2001), pp. 501-532.

[13] D. A. Winter, A. E. Patla, M. Ishac and W. H. Gage, "Motor mechanisms of balance during quiet standing”, J Electromyogr Kinesiol, vol. 13, no. 1, (2003) February, pp. 49-56.

[14] L. M. Nashner, “Adapting reflexes controlling the human posture”, Exp. Brain Res., vol. 26, (1976), pp. 5972.

[15] J. M. Macpherson, "Strategies that simplify the control of quadrupedal stance II: electromyographic activity", J. Neurophysiol., vol. 60, (1988), pp. 218-231.

[16] F. B. Horak and J. M. Macpherson, "Postural orientation and equilibrium", Handbook of Physiology. American Physiological Society, New York Section, vol. 12, (1996).

[17] S. M. Henry, J. Fung and F. B. Horak, "EMG responses to maintain stance during multidirectional surface translations”, J. Neurophysiol., vol. 80, (1998), pp. 1939-1950.

[18] S. B. Akaram, J. S. Frank, A. E. Patla and H. J. Alulum John, "Balance control during continuous rotational perturbation of the support surface”, Gait \& Posture, vol. 27, (2008), pp. 393-398.

[19] S. Corna, J. Tarantola, A. Nardone and A. Giordano, "Schieppati M. Standing on a continuously moving platform: is body inertia counteracted or exploited?”,Exp Brain Res, vol. 124, (1999), pp. 331-41.

[20] J. J. Buchanan and F. B. Horak, "Emergence of postural patterns as a function of vision and translation frequency”, Neurophysiol, vol. 81, (1999), pp. 2325-39.

[21] J. J. Buchanan and F. B. Horak, "Transitions in a postural task: do the recruitment and suppression of degrees of freedom stabilize posture?”, Exp Brain Res., vol. 139, (2001), pp. 482-94.

[22] S. B. Akaran, J. S. Frank, A. E. Patla and J. H. J. Allum, "Balance control during continuous rotational perturbations of the support surface”, Gate \& Posture, vol. 27, (2008), pp. 393-398.

[23] P. Gatev, S. Thomas, T. Kepple and M. Hallett, "Feedforward ankle strategy of balance during quiet stance in adults”, J Physiol, vol. 514(Pt 3), (1999), pp. 915-28.

[24] R. Johansson, M. Magnusson and M. Akesson, "Identification of human postural dynamics”, IEEE Trans Biomed Eng, vol. 35, no. 10, (1988) October, pp. 858-69.

[25] S. O. O'Sullivan, “Assessment of motor function”, O'Sullivan SB, Schmitz TJ, eds. Physical Rehabilitation: Assessment and Treatment, 4rd ed. Philadelphia, PA: FA Davis, (2001), pp. 501-532.

[26] O J. S., M. H. Lee, M. J. Lim and K. Y. Lee, “A Study of the Health Monitoring System for u- Healthcare”, The Journal of IWIT, vol. 9, no. 4, pp. 9-15, (2009).

[27] K. J. Lee and S. W. Park, "Patient Classification Scheme for Patient Information Management in Hospital UHealthcare System”, The Journal of IWIT, vol. 10, no. 3, (2010), pp. 131-137.

[28] D. A. Winter, A. E. Patla, F. Prince, M. Ishac and K. Gielo-Perczak, "Stiffness control of balance in quiet standing”, J Neurophysiol, vol. 80, (1998), pp. 1211-21.

[29] Y. Jian, D. A. Winter, M. G. Ishac and L. Gilchrist, "Trajectory of the body COG and COP during initiation and termination of gait”, Gait Posture, vol. 1, (1993), pp. 9-22.

[30] J. J. Buchanan and F. B. Horak, "Emergence of postural patterns as a function of vision and translation frequency”, J Neurophysiol, vol. 81, (2001), pp. 2325-39.

[31] Y. Ko, J. H. Challis and K. M. Newell, "Postural coordination patterns as a function of dynamics of the support surface”, Hum Mov Sci, vol. 20, (2001), pp. 737-64.

[32] J. J. Buchanan and F. B. Horak, "Transitions in a postural task: do therecruitment and suppression of degrees of freedom stabilize posture?”, Exp Brain Res., vol. 139, (2001), pp. 482-94. 
[33] J. J. Buchanan and F. B. Horak, "Vestibular loss disrupts control of head and trunk on a sinusoidally moving platform”, J Vestib Res., vol. 11, (2001), pp. 371-89.

[34] R. J. Peterka and P. J. Loughlin, "Dynamic regulation of sensori-motor integration in human postural control”, J Neurophysiol, vol. 91, (2004), pp. 410-23.

[35] J. H. J. Allum, F. Zamani, A. L. Adki and A. Ernst, "Differences between trunk sway characteristics on a foam support surface and on the Equitest1 ankle-sway-referenced support surface”, Gait Posture, vol. 16, (2002), pp. 264-70.

[36] R. Johansson, M. Magnusson and M. Akesson, "Identification of human postural dynamics”, IEEE Trans Biomed Eng, vol. 35, no. 10, (1988) October, pp. 858-69.

[37] P. Gatev, S. Thomas, T. Kepple and M. Hallett, "Feedforward ankle strategy of balance during quiet stance in adults”, J Physiol; vol. 514(Pt 3), (1999) February 1, pp. 915-28.

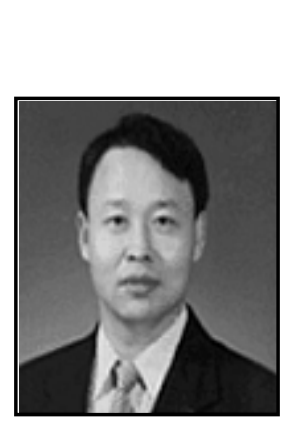

\section{Authors}

Jeong-Lae Kim received his B.S. degree in Bio-medical Engineering at Yonsei University in 1983, M.S. degrees in Electric Engineering at Konkuk University in 1989, and certificate program Ph.D. degrees in communication at KAIST in 1996, and Ph.D. degrees in biomedical Engineering at Yonsei University in 2011, South Korea. He joined Eulji University, South Korea, where he is now a professor in the department of Biomedical Engineering. He is a head of department of equipment management and bi-medical electronic instrument in BioMeditech Regional Innovation Center at Eulji University. He had worked in KOSOMBE (Korea Society Medical and Biological ), South Korea as a researcher of data information \& processing department from 1983 until now. His research interests include Medical image communication, Bio-physical signal communication, Bio-sensor system.

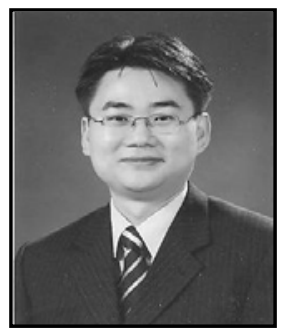

Kyu-Sung Hwang received his B.S. degree in Clinical Pathology at Yonsei University in 1998, M.S. degrees in Anatomy at Hanyang University in 2001, and Ph.D. degrees in Anatomy \& Cell biology at Hanyang University in 2007, South Korea. He joined Eulji University, South Korea, where he is now a professor in the department of Funeral Science. He is a dean of department of Funeral Science at Eulji University. He had worked in KPAA(The Korean Association of Physical Anthropologists) and Korean Association of Anatomists, South Korea as a researcher from 2001 until now. His research interests include Forensic Science, Anthropology, Embalming, Restorative art

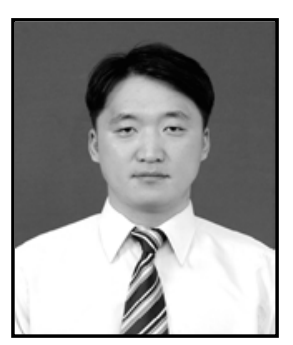

Yong-Seok Nam received bachelor's degree from the department of taekwodo at Korea National Sport University in 2001. Master's and doctorate degree from the department of Anatomy at Hanyang University College of Medicine in 2002, and in 2005. He was postdoctoral researcher and research professor from the department of Plastic Surgery at the Inha University College of Medicine in 20062008. He is currently working research professor from the department of Anatomy and the Catholic Institute for Applied Anatomy as a researcher and lecturer in the field of Gross Anatomy and Clinical Anatomy from 2008 until now. Research interests include Forensic Science, Anthropology, Embalming, Restorative art. 
International Journal of Bio-Science and Bio-Technology Vol.5, No.5 (2013) 